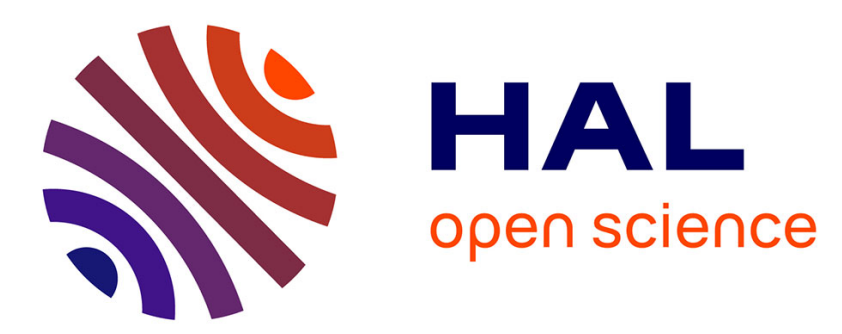

\title{
A Smit h-prediction based Haptic Feedback Controller for Time Delayed Virtual Environments Systems
}

\author{
Hichem Arioui, Said Mammar, Tarek Hamel
}

\section{To cite this version:}

Hichem Arioui, Said Mammar, Tarek Hamel. A Smit h-prediction based Haptic Feedback Controller for Time Delayed Virtual Environments Systems. American Control Conference, May 2002, Anchorage, United States. pp.4303-4309. hal-00702177

\section{HAL Id: hal-00702177 \\ https://hal.science/hal-00702177}

Submitted on 29 May 2012

HAL is a multi-disciplinary open access archive for the deposit and dissemination of scientific research documents, whether they are published or not. The documents may come from teaching and research institutions in France or abroad, or from public or private research centers.
L'archive ouverte pluridisciplinaire HAL, est destinée au dépôt et à la diffusion de documents scientifiques de niveau recherche, publiés ou non, émanant des établissements d'enseignement et de recherche français ou étrangers, des laboratoires publics ou privés. 


\title{
A Smith-prediction based Haptic Feedback Controller for Time Delayed Virtual Environments systems
}

\author{
H. Arioui, S. Mammar and T. Hamel \\ Complex Systems Laboratory - Evry University \\ 40, rue du Pelvoux, 91020 Evry Cedex, France \\ E-mail : arioui, smam, thamel@cemif.univ-evry.fr
}

\begin{abstract}
In this paper a modified Smith predictor for linear delayed haptic interaction is presented. The objective is to design a feedback law which compensates the time delay by its somehow elimination from the characteristic equation of the closed loop system. This method is robust within a certain safe prediction interval. The applicability of Smith predictor in the case of haptic interaction using computer haptic algorithms is shown.
\end{abstract}

\section{Introduction}

We are primarily concerned with stability analysis of systems that involves haptic devices used for interactive manipulations within virtual environments (VE). In figure $1 \mathrm{a}$ general schematic of such systems is shown.

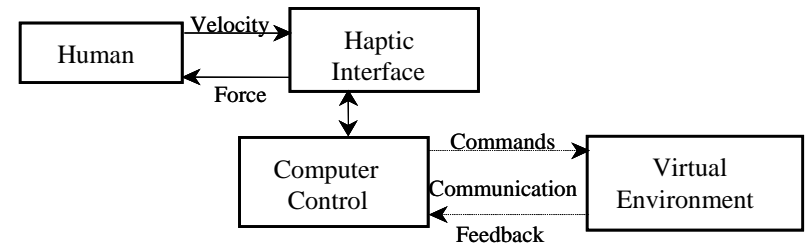

Figure 1: Haptic interaction system

VE haptic systems is similar with some extend to the well known master-slave teleoperation systems. Teleoperation systems synergistically combine humans and machines and this man-machine link is what makes them particular. In order to make the slave robot replicates operator desired trajectories in the one hand, and to convey the Kinesthetic slave robot-environment interaction as a feedback to the operator via the master robotic system in the other hand. A bilateral coupling control scheme which makes, in the ideal case, the master and the slave tracking each other is designed. As for teleoperation, VE haptics provide important information issued from synthetic virtual objects interactions within the VE. However, as for teleoperation, it is well known that small communication delays may destabilize the system using conventional bilateral con- trol methods. In the frame of teleoperation, Anderson and Spong [1] have proposed a control law for teleoperators with time delay based on passivity and scattering theory [2]. This method allows a stable behavior for any non-varying time delay. Niemeyer and Slotine [3] elegantly reformulate Anderson and Spong's work by introducing "wave variables" notion on the basis of network theory. The major problem when using wave variables still, up to date, the performances degradation as the delay increases. Several recent works extend the previous techniques for the time varying delay, as in the case of Internet [4], such as Stramigioli [5] and Yokokojhi [6] and for the enhancement of performances using a wave predictor [7].

Other approaches are based on the $H_{\infty}$ optimal control and $\mu$-synthesis, Leung [8]. Fiorini [9] analyzes the stability of a simple PD-type state feedback controller for a teleoperation system over Internet, using stability conditions derived from a proposed Lyapunov function.

For the use of Smith predictor to stabilize a linear time delayed system, several controller synthesis were presented. In [10], Smith and al. have proposed a delay compensation method which uses a model of the process in the feedback loop around a conventional controller. It is shown that using this technique the time delay can be eliminated from the characteristic equation of the system. Other approaches based on Smith predictor using state space equations have been proposed for treating the robustness towards initial conditions problems and disturbances [11] and [12]. Nevertheless, Smith predictor has never been implemented, from a pure control point-of-view, on time delayed teleoperation systems. This is due to the fact that it is not feasible to predict hazardous remote environments behavior. Indeed, it is difficult to actually predict when contact will occur, the contact interaction parameters when it occurs, the operator behavior and operator desired trajectories, etc. However, practical implementations have been made using various graphic predictors i.e. a VE (augmented reality based vision feedback, teleprogramming schemes, supervisory control, etc.) and discrepancies recovery strategies. 
The paper is organized as follows. We first define the model of the haptic interaction: a position-force based kinesthetic feedback scheme. We then briefly review the definition of Smith predictor and solutions for initial condition problems and disturbances problems. In the same section we discuss the applicability of the predictor in the case of haptic interaction. In section 4, we develop a controller in nominal case. Finally, simulation results on actual 1 DOF force feedback interface is presented and results about robustness of the control scheme are dropped.

\section{Previous Work in Haptic Simulation}

In Previous works on haptic systems has made the assumption that the operator and the virtual environment are passive, focusing on other aspects of the system to ensure stable haptic interaction. In fact, most of the proposed control strategies are typically various adaptations of the proposed bilateral controllers developed for force reflecting teleoperation systems. All these strategies are translated and adapted for the VE haptic interacts context mainly in a discrete form. Since Smith predictors have not been investigated for teleoperation systems, to the best authors knowledge, there seems to be no work addressing their implementation in the frame of VE haptics. Moreover, the limitations which inhibit the use of prediction for teleoperators do not hold in the VE haptics context since, contact prediction and involved virtual objects parameters are known thanks to the available computer haptics algorithms.

Adams and al. [13] presented an approach using the so called "virtual coupling" network between the haptic device and the held virtual object. The virtual coupling allows the environment design to be separated from the sampled-data control issues associated with haptic display. Though this solution has been already proposed in the context of teleoperation [14]. The main advantage of such a control design is that the parameterization of the virtual mechanism used for the coupling between the haptic interface and the manipulated virtual object can be elaborated so that the passivity of the whole system is preserved using linear interconnected networks theory.

Consider a one degree-of-freedom (DOF) haptic display shown in figure 2 with the haptic device $M(s)$, virtual coupling $C(z)$ and virtual environment $E(z)$.

If the condition :

$$
\operatorname{Re}\left(\frac{1}{C(z)}\right) \geq \frac{1-\cos (\angle Z O H(z))}{\operatorname{Re}(M(z))}|Z O H(z)|
$$

holds, the unconditional stability of the haptic interac-

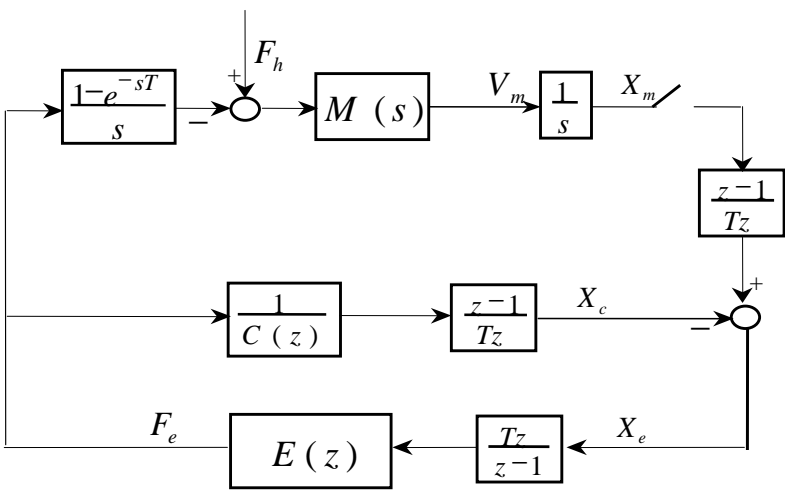

Figure 2: Model of one DOF haptic display

tion is satisfied. In other words, the haptic display appears passive, as seen by the operator [13]. The choice of the parameters of the virtual coupling should satisfy some performance properties.

If we interact with a remotely located VE (such as concurrent engineering design software or simply a shared VE) the communication channel is then located between the virtual coupling and the virtual environment as it is shown in the figure 1 . In this case, the communication time delay will indubitably destabilize the system since the passivity of the system is compromised and no longer preserved. One could compensate the time delay by a global reformulation of the controller using previously cited teleoperation force reflecting approaches. The solution proposed here, and formulated using Smith-based prediction, consists in keeping the virtual coupling as a local controller and compensates the communication time delay by a separate VE-based Smith prediction-type control. The detail of the controller design is explained in section 4 . In order to better understand the proposed method we will recall the Smith prediction principle .

\section{Smith prediction method}

Consider the following mono-variable system described by:

$$
\begin{gathered}
\left\{\begin{array}{l}
x(t)=A x(t)+B u(t-\tau) \\
y(t)=C x(t)
\end{array}\right. \\
x(0)=x_{0}, u(h)=u_{0}(h) \quad-\tau \leq h<0
\end{gathered}
$$

where $u$ is the control input, $x$ is the state variable, $y$ is the output, $\tau$ is the delay, and $u_{0}$ is the initial function of the input. $A, B$ and $C$ are constant matrices of appropriate dimensions for the process. It is assumed that $(A, B)$ is controllable and $(C, A)$ is observable. The transfer function of the closed loop system using a static unit feedback relating the output, $y(t)$, to the desired input $y_{d}(t)$ (see figure 3 ) is given 
by :

$$
\frac{Y(s)}{Y_{d}(s)}=\frac{C(s I-A)^{-1} B e^{-s \tau}}{1+C(s I-A)^{-1} B e^{-s \tau}}
$$

where $s$ is the Laplace transform variable. In this equation, note that the characteristic equation of the closed loop system contains the time delay element leading to an infinite number of eigenvalues. Controlling an infinite number of eigenvalues is not practically feasible. Using Smith prediction method the time delay element can be eliminated from the characteristic equation of the closed loop system. The design problem for the process with time delay can be transformed to the one without delay.

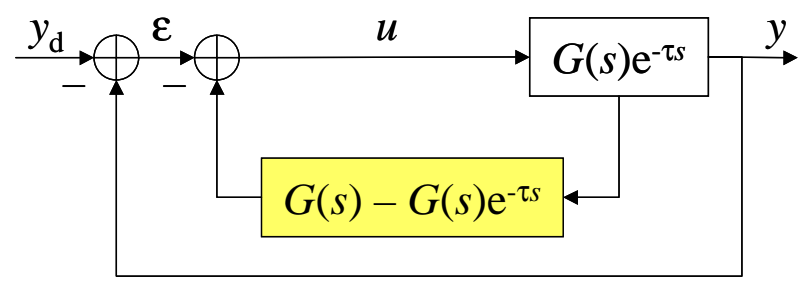

Figure 3: Smith predictor based control architecture

In figure $3, \varepsilon$ is the error, $y_{d}$ is the input signal, $y$ is the output system and $u$ is the control signal.

The Smith predictor control for system (2) is shown in figure 3. The block $G(s) e^{-s \tau}$, where $G(s)=C(s I-$ $A)^{-1} B$, is the process model without delay. The feedback element $G(s)-G(s) e^{-s \tau}$ is the nominal model of the process which is assuming that is well identified. The transfer function becomes :

$$
\frac{Y(s)}{Y_{d}(s)}=\frac{C(s I-A)^{-1} B e^{-s \tau}}{1+C(s I-A)^{-1} B}
$$

The system representing by the above transfer function is stable under some conditions on $A, B$ and $C$.

However, the Smith predictor controller cannot handle the disturbances and nonzero initial conditions as seen in [11]. A close analysis of many improved schemes (examples: Process-Model Control schemes [11], finite spectrum assignment techniques [12] show that they all use in an explicit or implicit manner a predictor of the state at time $t+\tau$ in order to achieve the control of the system (2). Unfortunately, in response to uncertain initial conditions or disturbances, the prediction cannot always ensure a stable system responses. This problem will not be treated in this paper (see [15] for more through development of the problem).

\section{Controller design based Smith predictor}

In this section, the Smith prediction of the previous example concerning system (2) is applied to the VE hap- tic reflecting system. The control scheme of the haptic interaction will contain a time delay between the virtual coupling and the virtual environment in both direction as presented in figure 1 by the communication channel. The transfer function of the closed loop simplified delayed system becomes in the continuous case:

the transfer function from $F_{h}$ to $F_{e}$ is given by

$$
\begin{gathered}
{\left[\left(F_{h}-F_{e} e^{-s \tau_{2}}\right) \frac{M(s)}{s}-F_{e} e^{-s \tau_{2}} C(s)\right] e^{-s \tau_{1}} E(s)=F_{e}} \\
\frac{F_{e}(s)}{F_{h}(s)}=\frac{\frac{1}{s} M(s) E(s) e^{-s \tau_{1}}}{1+e^{-s\left(\tau_{1}+\tau_{2}\right)} E(s)\left(\frac{1}{s} M(s)+C(s)\right)}
\end{gathered}
$$

It is stated clearly that the obtained closed loop (when contact is occurring because in the free motion the stability problem is not considered and the system is open loop) system has an infinite eigenvalues because the time delay element is present in the characteristic equation which may imply the potential instability of the system.

The proposed solution to this problem is a simple Smith predictor based controller using the process model of the haptic display performing like a feedback around the virtual environment. This is shown in figure 4 .

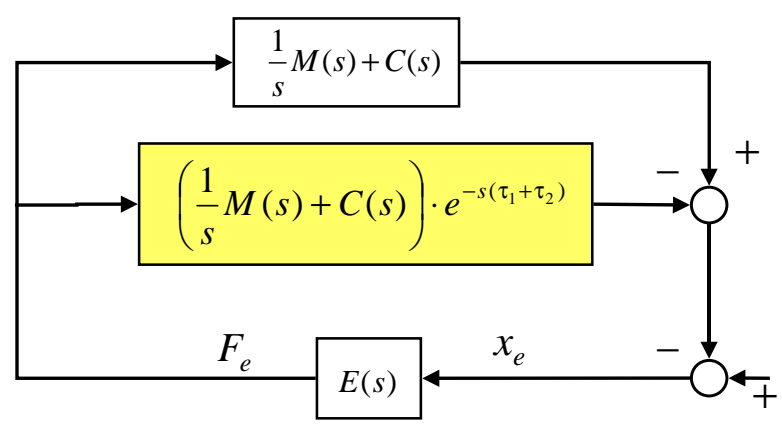

Figure 4: Controller design

The resulting transfer function of the global system is a stable haptic simulation with a delayed input

$$
\frac{F_{e}(s)}{F_{h}(s)}=\frac{\frac{1}{s} M(s) E(s) e^{-s \tau_{1}}}{1+E(s)\left(\frac{1}{s} M(s)+C(s)\right)}
$$

Note that there is no delay in the characteristic equation (3). The main advantage of this control-prediction scheme is the use of the process model of the haptic display only which is -in almost case- well know and its parameters are well identified. In other words, the use of the controller based on Smith prediction is to eliminate the delayed response $F_{e}$ of the master part and replace it with non-delayed response. The resultant global system is a system in which the delay is only in the force applied by the operator. 


\section{Simulation results}

\subsection{Nominal Design}

The haptic system simulation's characteristics are as follows:

- The master part is a single DOF haptic interface allowing displacements in a single direction. It is assumed that the haptic interface is a rigid stick of mass $m=0.2 \mathrm{Kg}$ mounted on a CC direct drive actuator for which we experiment different values of the viscous friction $\left(b=0.5 \frac{N . s}{m}\right.$ is a nominal values).

- The force $F_{e}$ is performed when a virtual contact occurs in the virtual environment (VE).

- The Virtual Coupling Network is chosen to be a PD-controller $C(s)=\frac{X_{c}(s)}{F_{e}(s)}=\frac{1}{B_{c} s+K_{c}}$, its role is to ensure unconditional stability under no time delay. $K_{c}=10 \frac{\mathrm{N}}{\mathrm{m}}$ and $B_{c}=10 \frac{\mathrm{N} . \mathrm{s}}{\mathrm{m}}$ are parameters which should satisfy condition (1).

- The VE is a virtual wall with stiffness coefficient $K_{e}=1000 \frac{N}{m}$. When collision occurs, the reflected force obeys to Hook's law, that is: $F_{e}=\left(x_{\text {wall }}-x_{e}\right) \times K_{e}=\Delta x \times K_{e}, x_{\text {wall }}$ is the position of the wall.

- Delays are taken to be $\tau_{1}=2 \tau_{2}=1 \mathrm{sec}$ (nominal values).

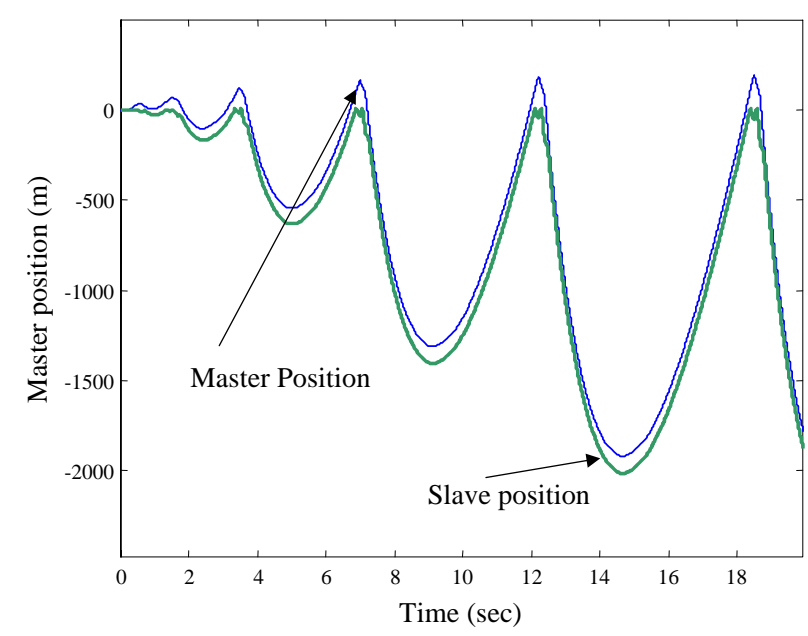

Figure 5: Unstable behavior of haptic simulation (multiple contact)

Simulation results are shown in figures 5, where the haptic simulation present a unstable behavior due to transmission delays $\left(\tau_{1}, \tau_{2}\right)$.

Now, we apply the developed prediction-based controller (with nominal parameters), we obtain as expected from theory a stable simulation with position error when contact is occurs. The position error (in constrained motion) between master and slave is inversely proportional to viscous friction coefficient of the haptic interface $b$ and delay $\tau_{2}$ in control $F_{e}$. In other words, the collision detection in the VE and the execution of the command due to the collision the operator continue to move. In the free motion, the slave position is equal to master delayed position (delay equal to $\tau_{1}$ ) figure 6 .

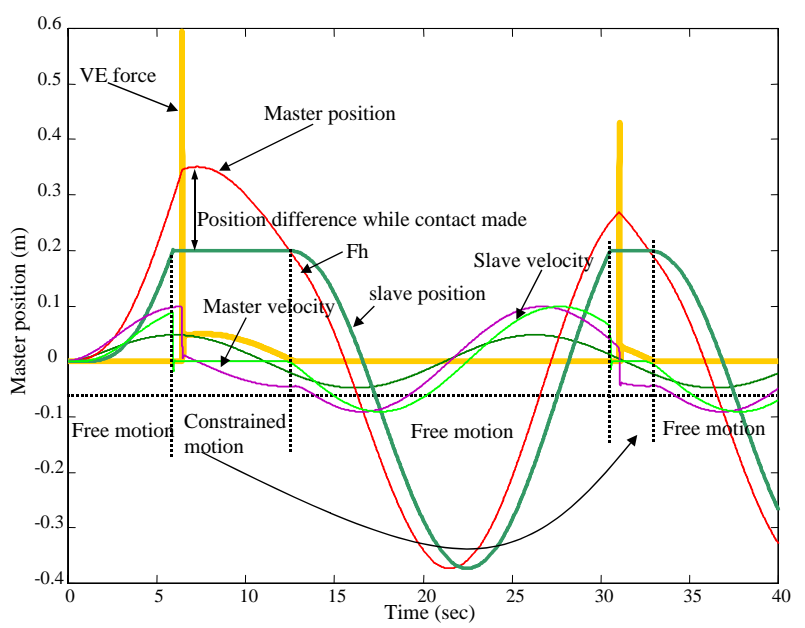

Figure 6: Stabilization with Smith-predictor Controller

Figure 6 shows the master position and the progressive attenuation of the unavoidable discrepancy (whatever the controller is) of the master and virtual positions during the contact. The explanation is quite simple : At the beginning, the master speed increases as the applied operator force $\left(F_{h}\right)$ increases. When the contact is made $\left(v_{m}=0.1 \mathrm{~m} \mathrm{sec}-1, x_{m}=0.34 m\right)$, we can notice that the master speed drops toward zero, then continue to decrease. Because of the operator speed and the backward delay, the error (the penetration) is exactly $\epsilon=0.007 \mathrm{~m}$ for the first contact, which means that the operator feels the force of the VE $\left(F_{e}\right)$ when $x_{m}=0.34 m$. The constraint on slave position is respected $(0.2 m$ on the figure 6$)$ due to the high stiffness of the virtual wall. The VE reaction force $\left(F_{e}\right)$ is well fed and counterparts, to some extent, the applied $F_{h}$. The latter increases to a maximum and decreases, this behavior is desired, as we took a sin function for $F_{h}$. One can notice the good fidelity in force and velocity restitution (as there is no error between the master and slave velocities during free motions). When the contact brakes, the remote position matches the desired one after a delay $\tau_{1}$. Several simulations where performed with various contact behavior (multiple contacts, and various VE stiffness), the results show a good stable behavior of the haptic interface. 


\subsection{Robustness and limitations of the control scheme}

A future work will contain through developments and explanation concerning the robustness issues due to error estimation of the master model and we have extend this method which became free from the delay estimation. In the allowed space we only present some results concerning system behavior due to errors in the estimation in the delay value.

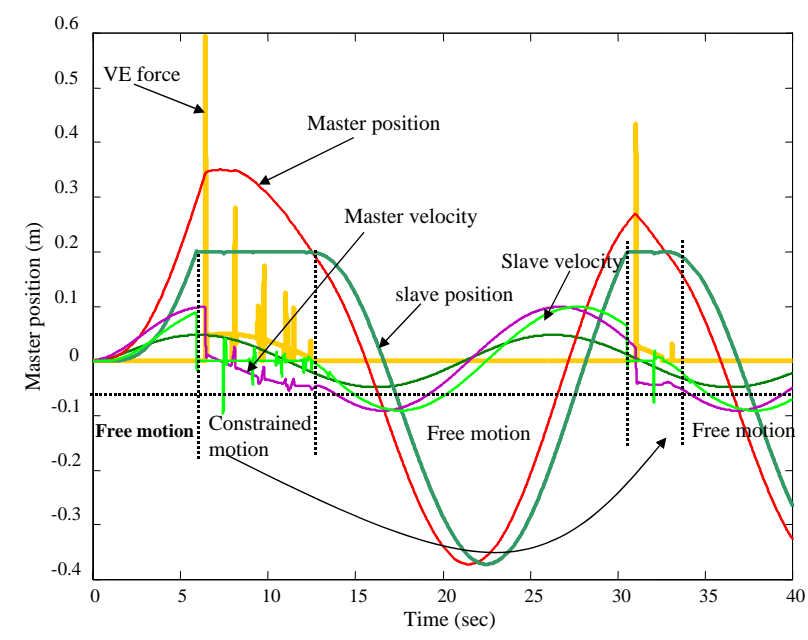

Figure 7: Robustness of controller du to mismatch delays $\Delta \tau=50 \mathrm{~ms}$

Figure 7 shows a stable behavior of the haptic interaction when $\left.\left.\Delta \tau=\Delta \tau_{1}+\Delta \tau_{2} \in\right] 0,0.05\right] s$ (where $\Delta \tau_{i}$ is the error estimation between the transmission delay $\tau_{i}$ and the virtual delay used by the Smith controller), however, it can be noticed that small oscillations appear in virtual environment force or velocities which may deteriorate system performances. It should be noted, that same oscillations appear when error estimation in model of the haptic interface is considered.

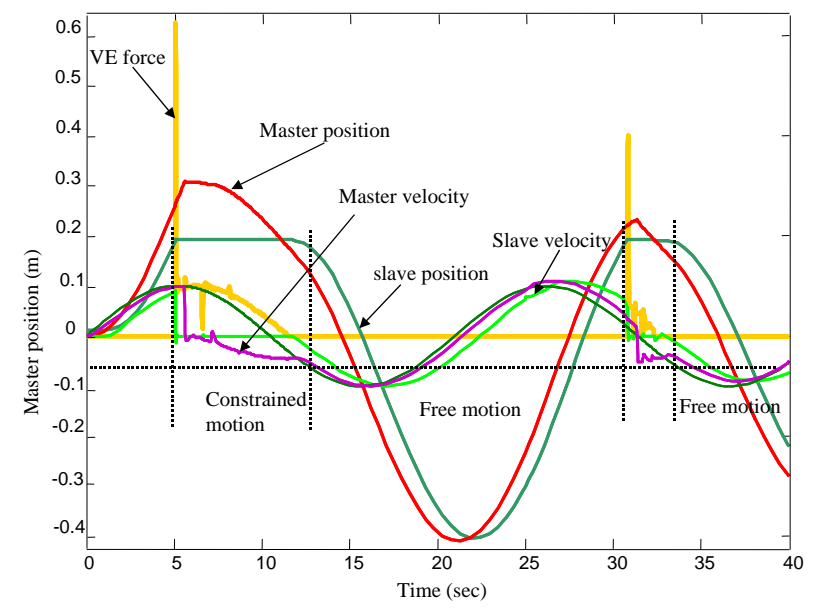

Figure 8: Robustness of controller due to mismatch in model estimation $m=0.045 \mathrm{~kg}$

It should be noted, that same oscillations appear when error estimation in model of the haptic interface is considered. Figure 8 shows a stable behavior due to perturbations in model estimation of haptic interface using in Smith predictor Controller, considering errors only in masse, the margin giving by Rouche's Theorem is $|\Delta m| \leq 45 g$ (see [?] for more through development of the robustness problem).

\section{Conclusion}

In this paper, a controller stabilising delayed haptic feedback system and force feedback teleoperators has been presented. The originality and the main idea of this work is the investigation of a Smith-like prediction within the remote site (i.e. slave robot site for teleoperation case, or in the simulation engine within remote VE). The proposed controller has been proven to stabilize force feedback interaction for important delays and different master to remote transmission delays. Only the model of the master haptic interface is needed, and in most cases this model is available. Indeed the controller guarantees the stability of the system but generates a none desirable static tracking error. The controller is also robust to small system parameters fluctuations. The development of the solution to the static error with a thorough robustness analysis are presented in another companion paper.

\section{Acknowledgment}

We would like to express our gratitude to Habiba DALI for fruitful discussion and her contribution.

\section{References}

[1] R.J. Anderson and M.W. Spong, "Bilateral control of teleoperators with time delay", IEEE Transactions On Automatic Control, vol. 34, no. 5, pp. 494501, May 1989.

[2] C.A. Desoer and M. Vidyasagar, "Feedback Systems: Input-Output Properties", Academic Press, 1975 .

[3] G. Niemeyer and J.J. Slotine, "Using Wave Variables for system Analysis and Robot Control", IEEE International Conference on Robotics and Automation, pp. 1619-1625, April 1997, Albuquerque, New Mexico.

[4] G. Niemeyer and J.J. Slotine, "Towards forceReflecting Teleoperation over the Internet", IEEE International Conference on Robotics and Automation, pp. 1909-1915, May 1998, Leuven, Belgium.

[5] S. Stramigioli, A. V.D. Schaft, and B. Maschke, "Geometric Scattering in tele-manipulation of port controlled Hamiltonian systems", In Proceedings of the 
39th IEEE Conference on Decision and Control, 2000, Sydney, Australia.

[6] Y. Yokokojhi, T. Imaida, and T. Yoshikawa, "Bilateral Control with Energy Balance Monitoring Under Time-Varying Communication Delay", IEEE International Conference on Robotics and Automation, pp. 2434-2439, April 2000, San Francisco, CA.

[7] M. Saghir and W.J. Book, "Wave-Based Teleoperation with Prediction", In Proceedings of the American Control Conference, pp. 4605-4611, June 2001, Arlington, VA.

[8] G.M.H. Leung, B.A. Francis, and J. Apkarian, "Bilateral Controller for Teleoperators with Time Delay via $\mu$-Synthesis", IEEE International Conference on Robotics and Automation, vol. 11, no. 1, pp. 105116, February 1995.

[9] R. Oboe and P. Fiorini, "A Design and Control Environment for Internet-Based Telerobotics", The International Journal of Robotics Research, vol. 17, no. 4, pp. 433-449, April 1998.

[10] O.J.M. Smith, "A Controller to overcome dead time", ISA J., vol. 6, no. 2, pp. 28-33, 1959.

[11] K. Watanabe and M. Ito, "A Process-Model Control for Linear System with Delay", IEEE Transactions on Automatic Control, vol. AC-26, no. 6, pp. 12611268, December 1981.

[12] A. Manitius and W. Olbrot, "Finite Spectrum assignment problem for systems with delays", IEEE Trans. on Auto. Control, vol. AC-24, pp. 541-553, August 1979.

[13] R.J. Adams and B. Hannaford, "Stable Haptic Interaction with Virtual Environments", IEEE Trans. Robot. Automat., vol. 15, no. 3, pp. 465-474, June 1999.

[14] C. Andriot and L.Joly, "Imposing Motion Constraints to a Force Reflecting Telerobot through RealTime Simulation of Virtual Mechanism", IEEE Int. Conf on Robotics and Automation, vol. 1, pp. 357-362, 1995, Nagoya, Japan.

[15] S. Mondié, M. Dambrine, and O. Santos, "Approximation of control laws with distributed delays: a necessary condition for stability", IFAC Conference on Systems, Structure and Control, August 2001, Prague, Czech Republic. 